\title{
Heat shock protein 70 induction by glutamine increases the $\alpha$-synuclein degradation in SH-SY5Y neuroblastoma cells
}

\author{
JIA YANG $^{1}$, YANMEI ZHANG ${ }^{2}$, SHIGANG ZHAO $^{3}$, ZHELIN ZHANG $^{3}$, \\ XIUQING TONG ${ }^{3}$, FANG WEI ${ }^{3}$ and ZUNENG LU ${ }^{1}$ \\ ${ }^{1}$ Department of Neurology, Renmin Hospital of Wuhan University, Wuhan, Hubei 430060; \\ ${ }^{2}$ Department of Neurology, The People's Hospital of Inner Mongolia, Hohhot, Inner Mongolia 010055; \\ ${ }^{3}$ Department of Neurology, Affiliated Hospital of Inner Mongolia Medical University, \\ Hohhot, Inner Mongolia 010059, P.R. China
}

Received August 24, 2014; Accepted June 5, 2015

DOI: $10.3892 / \mathrm{mmr} .2015 .4027$

\begin{abstract}
Functional defects in heat shock proteins (HSPs), e.g. Hsp70, have been reported to have a key role in Parkinson's disease (PD). Overexpressed Hsp70 re-folds aggregated $\alpha$-synuclein to generate the non-toxic and non-aggregated form. Thus, Hsp70 is a well-defined therapeutic target, and Hsp70 promotion is an efficient strategy to prevent or even reverse the $\alpha$-synuclein-induced toxicity in PD. The present study investigated the promotion of Hsp70 expression in SH-SY5Y neuroblastoma cells by glutamine (Gln), which has recently been recognized to induce Hsp70 expression. Furthermore, the role of heat shock factor (HSF)-1 in the Gln-mediated upregulation of Hsp70 expression was investigated. In addition, the regulatory role of Gln in $\alpha$-synuclein degradation in $\alpha$-synuclein-overexpressing SH-SY5Y cells was determined. The results of the present study demonstrated that Gln treatment significantly upregulated Hsp70 expression at the mRNA as well as the protein level in a dose-dependent and time-dependent manner. Gln-induced Hsp70 upregulation was found to be HSF-1-dependent, as HSF-1 knockdown abrogated the Hsp70 upregulation by Gln in $\alpha$-synuclein-overexpressing SH-SY5Y cells. In conclusion, present study confirmed that Gln upregulates Hsp70 expression in SH-SY5Y neuroblastoma cells in an HSF-1-dependent manner. The upregulation of Hsp70 by Gln increases the $\alpha$-synuclein degradation. Therefore, Gln may be a potential therapeutic agent to prevent $\alpha$-synuclein aggregation in PD.
\end{abstract}

Correspondence to: Dr Zuneng Lu, Department of Neurology, Renmin Hospital of Wuhan University, 238 Jiefang Road, Wuhan, Hubei 430060, P.R. China

E-mail: zuneng_luwh@163.com

Key words: heat shock protein 70, glutamine, $\alpha$-synuclein degradation, neuroblastoma cells

\section{Introduction}

Parkinson's disease (PD) is one of the most common neurodegenerative disorders (1), with prominent characteristics including the degradation of dopaminergic cells within the substantia nigra pars compacta (2), aberrant intracellular protein aggregation in the dorsal motor nucleus of the vagus, a region within the medulla oblongata $(3,4)$. These aggregates are known as Lewy bodies, with $\alpha$-synuclein ( $\alpha$-Syn) as the major component (5). Striking evidence has confirmed that $\alpha$-Syn has a key role in the formation and progression of PD; however, the mechanism underlying the cytotoxicity of $\alpha$-Syn in PD remains to be determined $(6,7) . \alpha$-Syn is a $14-\mathrm{KDa}$ neuronal protein, belonging to a family of structurally associated proteins in the brain $(8,9)$. Under physiological conditions, $\alpha$-Syn is highly expressed at pre-synaptic terminals and promotes the assembly of the SNARE machinery (10), with an importance for neurotransmitter release (11) and the protection of nerve terminals against injury (12). $\alpha$-Syn has been widely accepted to have a natively unfolded tertiary structure as its main physiological form in the brain (13). It is the key pathological course for $\alpha$-Syn aggregation to proceed from monomers to pathogenic protein inclusions (14).

Aggregation of $\alpha$-Syn monomers leads to the formation of soluble oligomeric species, which, according to in vitro experiments, further spontaneously aggregate in the absence of other proteins, such as molecular chaperones (15). The accumulation and aggregation of $\alpha$-Syn in PD may reflect changes to its synthesis and/or degradation (16). Besides an increased $\alpha$-Syn gene copy number (17) supporting the role of increased $\alpha$-Syn synthesis in $\mathrm{PD}$, there is increasing evidence that the impaired degradation pathways of $\alpha$-Syn may also be compromised in PD $(18,19)$. The importance of molecular chaperones has also been underlined by the fact that overexpression of these molecules, such as heat shock proteins (HSPs), leads to the re-folding of aberrant $\alpha$-Syn aggregates to form non-toxic and non-aggregated $\alpha$-Syn $(20,21)$. Therefore, functional defects of HSPs may have a key role in PD $(21,22)$. Hsp70 is the most recognized molecular chaperone, and has been linked with PD and $\alpha$-Syn aggregation. Studies have confirmed the negative regulatory role of Hsp70 in $\alpha$-Syn aggregation in PD and in 
$\alpha$-Syn-induced toxicity in cells $(22,23)$. Therefore, Hsp70 is a well-defined therapeutic target, and the upregulation of Hsp70 is an efficient strategy to block or even reverse $\alpha$-Syn-induced toxicity in PD.

The present study investigated the upregulation of Hsp70 expression in SH-SY5Y neuroblastoma cells by glutamine (Gln) and assessed the role of heat shock factor (HSF)-1 in this process. Furthermore, the regulatory role of Gln in the $\alpha$-Syn degradation in $\alpha$-Syn-overexpressing SH-SY5Y cells was investigated. The results of the present study indicated that glutamine may be a potential therapeutic agent to prevent $\alpha$-Syn aggregation in PD.

\section{Materials and methods}

Reagents, cell culture and treatments. L-Gln was purchased from Sigma-Aldrich (St. Louis, MO, USA). The SH-SY5Y human neuroblastoma cell line was obtained from the Type Culture Collection of the Chinese Academy of Sciences (Beijing, China) and was cultured in Dulbecco's modified Eagle's medium (DMEM; Invitrogen Life Technologies, Carlsbad, CA, USA) supplemented with 10\% FBS (Invitrogen Life Technologies) or maintained in DMEM supplemented with $2 \%$ FBS. To generate $\alpha$-Syn-overexpressing SH-SY5Y cells [SH-SY5Y (Syn+)], an $\alpha$-Syn coding sequence was amplified using Phusion High-Fidelity DNA Polymerase (New England Biolabs, Beverly, MA, USA) with the following primers: Forward, 5'-CGCGACGCGGAAGTGAGGTGC-3' and reverse, 5'-TTCTGGGCTACTGCTGTCAC-3', and subsequently cloned into the pcDNA3.1(+) eukaryotic expression vector (cat. no. V790-20; Thermo Fisher Scientific, Inc., Rockford, IL, USA). Following transfection with the recombinant pcDNA3.1- $\alpha$-Syn or pcDNA3.1-CAT plasmid (cat. no. V790-20; Thermo Fisher Scientific, Inc.) using Lipofectamine 2000 (Invitrogen Life Techonologies), SH-SY5Y cells were cultured for three passages in the presence of $800 \mu \mathrm{g} / \mathrm{ml} \mathrm{G} 418$ (Life Technologies, Grand Island, NY, USA) to select the positive $\alpha$-Syn-overexpressing clones, SH-SY5Y (Syn+), which were maintained in the presence of $500 \mu \mathrm{g} / \mathrm{ml} \mathrm{G} 418$. The HSF-1-specific small interfering (si)RNA (with corresponding siRNA sequences as follows: Forward, 5'-GAA CGA CAG UGG CUC AGC AUU-3' and reverse, 5'-P-UGC-UGA GCC ACU GUC GUU CUU-3') or control siRNA (with scrambled siRNA sequences as follows: Forward, 5'-GUA ACU GCA ACG AUU UCG AUG DTDT-3' and reverse, 5'-CAU CGA AAU CGU UGC AGU UAC DTDT-3'; Sangon, Shanghai, China) was transfected into the SH-SY5Y (Syn+) cells with Lipofectamine 2000 at 25 or $50 \mathrm{nM}$ to knock down HSF-1 expression.

$R N A$ isolation and reverse transcription quantitative polymerase chain reaction $(R T-q P C R)$. Total cellular RNA was isolated using TRIzol (Invitrogen Life Technologies) according to the manufacturer's instructions, and was supplemented with RNase inhibitor (New England Biolabs). The expression of Hsp70, HSF-1 and $\alpha$-Syn mRNA was quantified using the real-time RT-qPCR method. cDNA was synthesized using the Quanti Tect Reverse Transcription kit (Qiagen, Valencia, CA, USA). qPCR was performed using a SYBR PrimeScript RT-qPCR kit (TaKaRa Bio, Inc., Tokyo, Japan) with the following primers: Forward, 5'-AGG ACT TTC AAA GGC CAA GG-3' and reverse, 5'-TCC TCC AAC ATT TGT CAC TTGC-3' for $\alpha$-Syn; forward, 5'-TGT GTC TGC TTG GTA GGA ATG GTG GTA-3' and reverse, 5'-TTA CCC GTC CCC GAT TTG AAG AAC-3' for HSP70; forward, 5'-CGA CAG TGG CTC AGC ACA TTC C-3' and reverse, 5'-CAG CTC GGT GAT GTC GGA GAT G-3' for HSF-1; and forward, 5'-TGT CCA CCT TCC AGC AGA TGT-3' and reverse, 5'-AGC TCA GTA ACA GTC CGC CTA GA-3' for $\beta$-actin ( $\beta$-actin served as an internal control). mRNA samples were amplified using primer sets specific for the target gene on a Lightcycler 480 II (Roche Diagnostics, Basel, Switzerland). Relative quantification was performed using the $\Delta \Delta \mathrm{Ct}$ method using $\beta$-actin as the reference gene (24).

Western blot analysis. SH-SY5Y or SH-SY5Y (Syn+) cells were washed with cold phosphate-buffered saline (PBS) and then lysed using NE-PER ${ }^{\mathrm{TM}}$ Nuclear and Cytoplasmic Extraction Reagents (cat. no. 78833; Thermo Fisher Scientific). Protein samples were supplemented with protease inhibitor cocktail (cat. no. 04693116001, Roche Diagnostics) and quantified using a bicinchoninic acid protein assay reagent (cat. no. 23234; Pierce Biotechnology, Inc., Rockford, IL, USA), and were separated using 10\% gradient SDS-PAGE. The separated proteins were then transferred onto a polyvinylidene difluoride membrane, which was blocked in 5\% skimmed milk. The membranes were then incubated with the primary antibody for $1 \mathrm{~h}$ at room temperature or overnight at $4^{\circ} \mathrm{C}$, followed by the secondary horseradish peroxidase-conjugated anti-rabbit antibody for $1 \mathrm{~h}$ at room temperature. The membranes were washed three times with PBS before each inoculation with the primary or secondary antibodies. Target proteins were visualized using an enhanced chemiluminescence detection system (RPN 2106; Amersham Pharmacia Biotech, Amersham, UK) and analyzed with ImageJ software (http://rsb.info.nih.gov/ij/). Rabbit polyclonal antibodies to $\alpha$-Syn (cat. no. 2642S; 1:300; Cell Signaling Technology Inc., Danvers, MA, USA) Hsp70 (cat. no. 4872S; 1:200; Cell Signaling Technology Inc.) or $\beta$-actin (cat. no. A2066; 1:500; Sigma-Aldrich) and rabbit polyclonal antibodies to HSF-1 (cat. no. sc-9144; Santa Cruz Biotechnology, Dallas, TX, USA) were used to quantify the expression of the target proteins.

Statistical analysis. Statistical analysis was performed with GraphPad Prism 6 (GraphPad Software, Inc., La Jolla, CA, USA). Differences in the mRNA or protein expression of $\alpha$-Syn, Hsp70 or HSF-1 between two groups were analyzed using Student's t-test. All values are expressed as mean \pm standard error. $\mathrm{P}<0.05$ was considered to indicate a statistically significant difference between values.

\section{Results}

Gln upregulates Hsp70 expression in SH-SY5Y neuroblastoma cells. Previous studies have shown that Gln safely enhances HSP expression in in vitro and in vivo settings (25-28). Given the key regulatory role of Hsp70 in $\alpha$-synuclein degradation, which is deregulated in $\mathrm{PD}$, the present study investigated the possible regulation of Hsp70 expression by Gln in SH-SY5Y neuroblastoma cells. Hsp70 mRNA expression levels in 
A

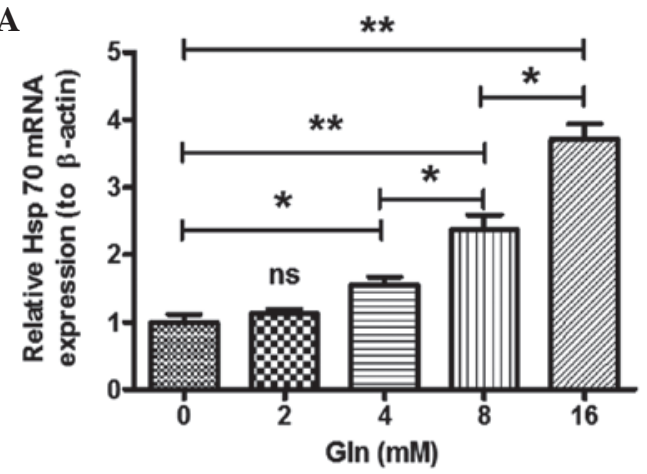

C
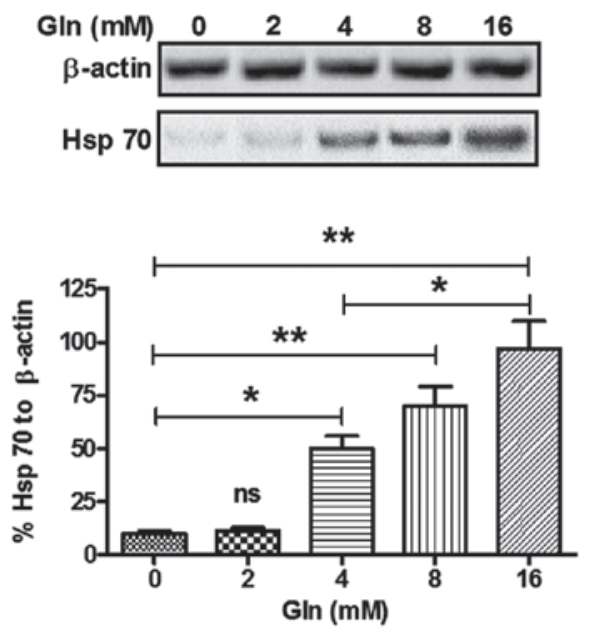

B

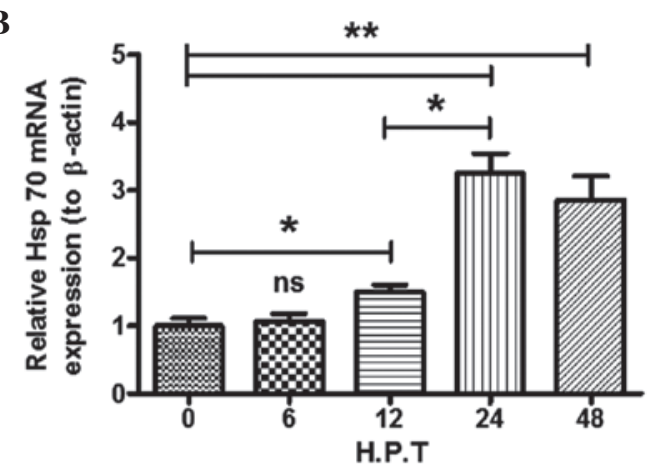

D

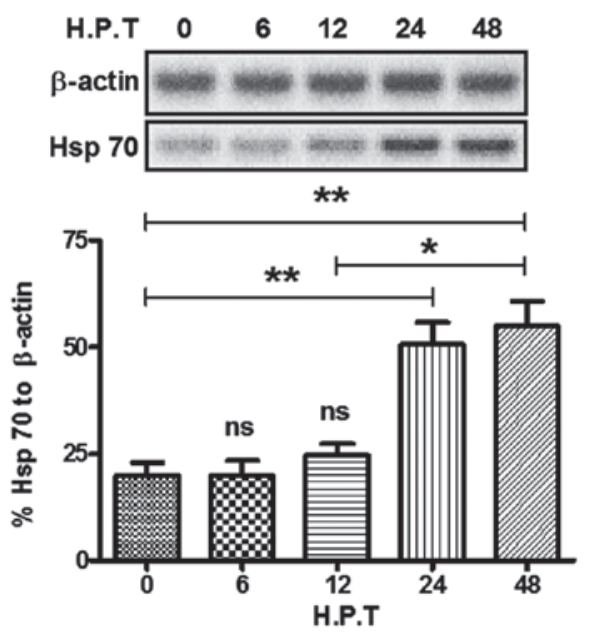

Figure 1. Glutamine treatment upregulates Hsp70 expression in SH-SY5Y neuroblastoma cells. (A) Hsp70 mRNA levels in SH-SY5Y cells post-Gln treatment at various concentrations $(0,2,4,8$ or $16 \mathrm{mM})$ for $24 \mathrm{~h}$; (B) Hsp70 mRNA levels in SH-SY5Y cells post-Gln treatment (4 mM) for various durations [0, 6, 12 , 24 or 48 h post treatment (HPT)]; (C) Western blot analysis of Hsp70 in SH-SY5Y cells post-Gln treatment (0, 2, 4, 8 or 16 mM) for 24 h. HSP70 levels were normalized to $\beta$-actin. (D) Hsp70 protein expression in SH-SY5Y cells post-Gln treatment (4 mM) for various durations (0, $6,12,24$ or 48 HPT). Values are expressed as the mean \pm standard error of triple experiments. ${ }^{*} \mathrm{P}<0.05,{ }^{* *} \mathrm{P}<0.01$. ns, no significance; HSP, heat shock protein; gln, glutamine.

SH-SY5Y cells post Gln treatment were determined using RT-qPCR. Fig. 1A shows that Gln treatment (4-16 $\mathrm{mM})$ for $24 \mathrm{~h}$ significantly upregulated Hsp70 mRNA expression $(\mathrm{P}<0.05$ for $4 \mathrm{mM}, \mathrm{P}<0.01$ for $8 \mathrm{mM}$ and $\mathrm{P}<0.01$ for $16 \mathrm{mM})$. Of note, the Gln-mediated upregulation of Hsp70 expression was dose dependent, as there was a significant difference in Hsp70 mRNA levels between the 4 and $8 \mathrm{mM}$ Gln groups, as well as between the 8 and $16 \mathrm{mM}$ Gln groups. The time-dependence of the Hsp70 upregulation by Gln was also confirmed; Fig. 1B indicates that Hsp70 mRNA was upregulated at $12 \mathrm{~h}$ post-Gln treatment $(4 \mathrm{mM})$ and peaked $24 \mathrm{~h}$ later, and there was a significant difference in Hsp70 mRNA levels between cells treated for $12 \mathrm{~h}$ and cells treated for $24 \mathrm{~h}(\mathrm{P}<0.05)$. To reconfirm the upregulation, the Hsp70 protein levels in SH-SY5Y cells post-Gln treatment were also examined. Fig. $1 \mathrm{C}$ and D show that Gln treatment at $4-16 \mathrm{mM}$ for $24 \mathrm{~h}$ or at $4 \mathrm{mM}$ for 24-48 h also promoted the protein expression of Hsp70 in a dose- and time-dependent manner $(\mathrm{P}<0.05$ or $\mathrm{P}<0.01)$.

Upregulation of Hsp70 by Gln in SH-SY5Y cells is HSF-1-dependent. In previous studies, HSF has been confirmed to bind to a target sequence, the heat shock element (HSE), located in the promoters of heat-induced genes and promote the expression of HSPs, including Hsp70 (29-31). In order to identify the signaling pathways of Gln-induced upregulation of Hsp70 expression in SH-SY5Y cells, the present study investigated the effect of HSF-1 knockdown on Gln-induced Hsp70 expression. RNA interference technology was adopted to knockdown HSF-1 expression, and the results shown in Fig. 2A demonstrated that HSF-1-specific siRNA, siRNA-HIF-1, significantly downregulated the HSF-1 mRNA expression $(\mathrm{P}<0.05$ for $25 \mathrm{nM}$ and $\mathrm{P}<0.01$ for $50 \mathrm{nM})$ in $\mathrm{SH}-\mathrm{SY} 5 \mathrm{Y}$ cells following Gln treatment ( $8 \mathrm{mM}$ for $24 \mathrm{~h}$ ). HSF-1 protein levels were also downregulated following siRNA-HSF-1 transfection $(\mathrm{P}<0.05$ or $\mathrm{P}<0.01$, respectively; Fig. $2 \mathrm{~B}$ and $\mathrm{C})$. Furthermore, the Hsp70 was also significantly downregulated at the protein $(\mathrm{P}<0.05$; Fig. 2B and $\mathrm{C})$ and mRNA $(\mathrm{P}<0.05$ for $25 \mathrm{nM}$ and $\mathrm{P}<0.01$ for $50 \mathrm{nM}$; Fig. $2 \mathrm{~B}$ and $\mathrm{C}$ ) level, compared to that in the siRNA control-transfected cells. These results confirmed that the Gln-induced Hsp70 expression was HSF-1-dependent.

$\alpha$-Syn overexpression in SH-SY5Y cells has no influence on Hsp70 and HSF-1 expression. Functional defects of HSPs are thought to have a key role in PD $(21,22)$. Hsp70 is the most investigated molecular chaperone and is known to negatively regulate $\alpha$-Syn aggregation in PD and to mediate $\alpha$-Syn-induced toxicity in cells $(22,23)$. To explore the influence of Hsp70 on $\alpha$-Syn degradation following Gln-induced Hsp70 upregulation, the 

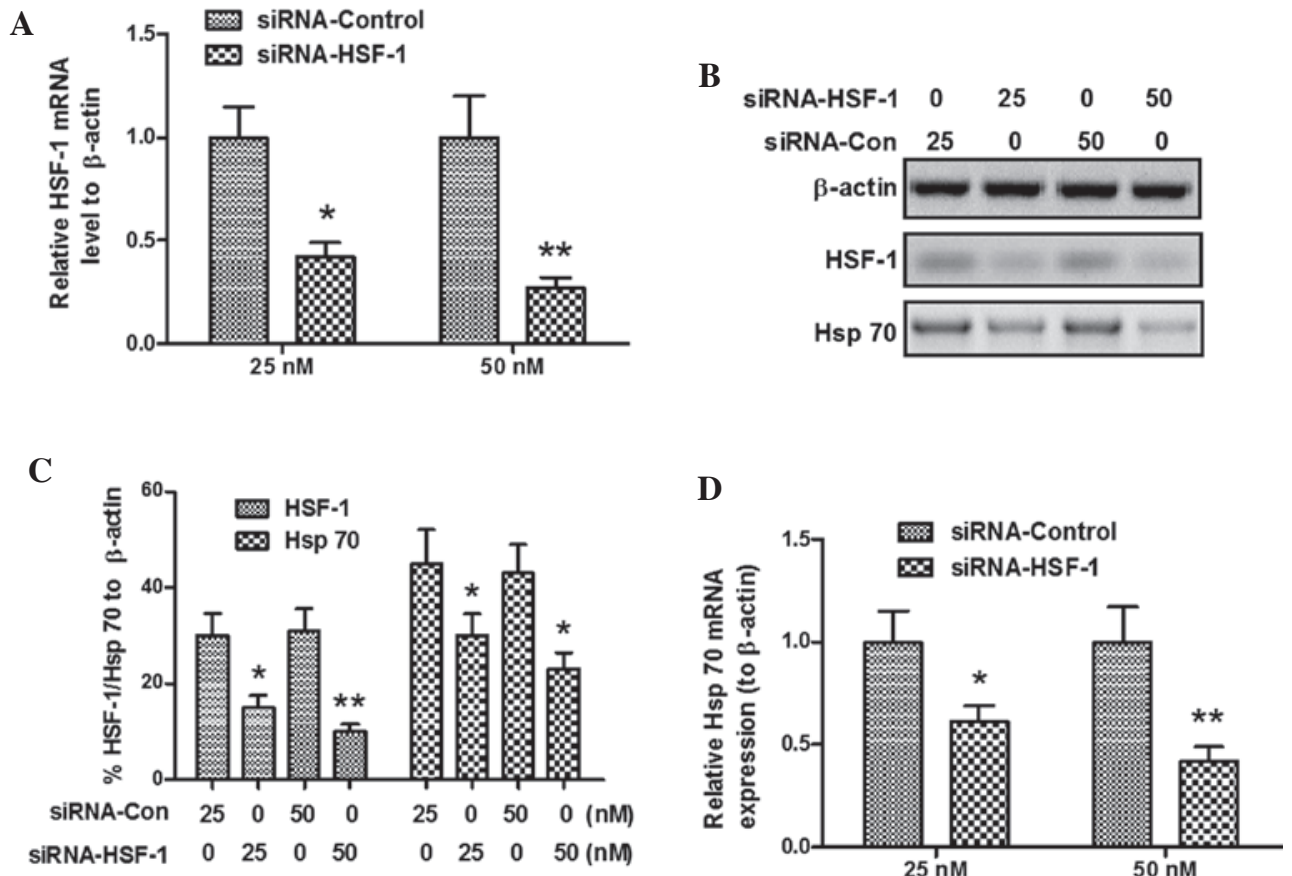

Figure 2. HSF-1 silencing by siRNA abrogates Gln-mediated Hsp70 upregulation. (A) HSF-1 mRNA was significantly downregulated by the transfection of HSF-1-targeted siRNA at a concentration of 25 or $50 \mathrm{nM}$. siRNA-Con was used as a control siRNA; (B) Western blot analysis of the protein levels of HSF-1 and HSP70 following HSF-1-targeted siRNA transfection; (C) siRNA-HSF-1 blocked the Gln-mediated increases of HSF-1 and HSP70 protein levels; (D) The Gln-mediated upregulation of HSP70 mRNA levels was abrogated following siRNA-HSF-1 transfection. All SH-SY5Y cells in this experiment were cultured with medium supplemented with $4 \mathrm{mM}$ Gln, and all experiments were performed in triplicate. Values are expressed as the mean \pm standard deviation of triple experiments. ${ }^{*} \mathrm{P}<0.05 ;{ }^{* *} \mathrm{P}<0.01$ vs. control. HSP, heat shock protein; HSF, heat shock factor; Gln, glutamine; siRNA, small interfering RNA; Con, control.

A

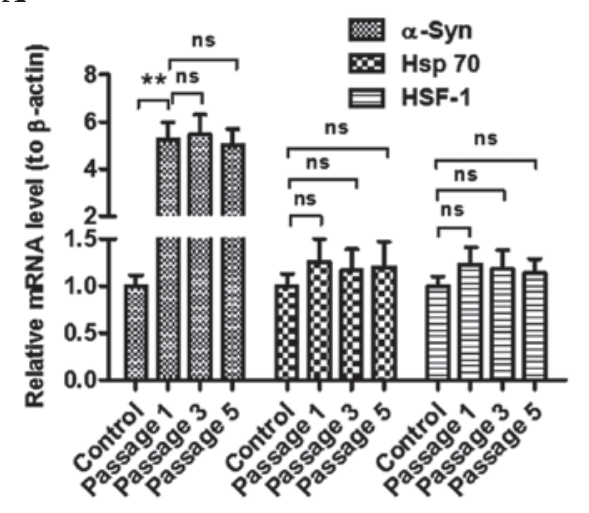

C

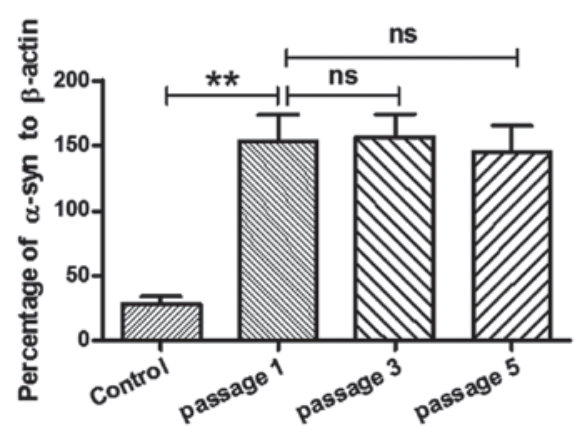

B

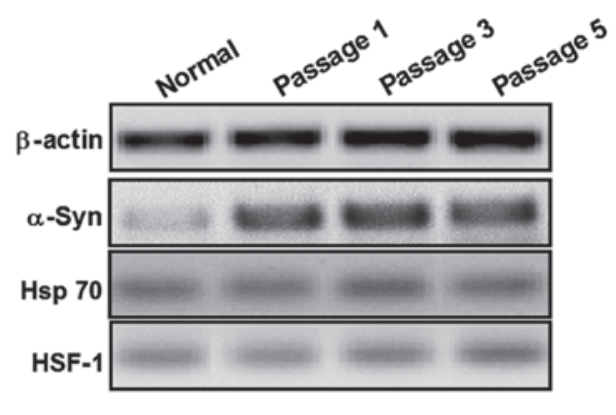

D

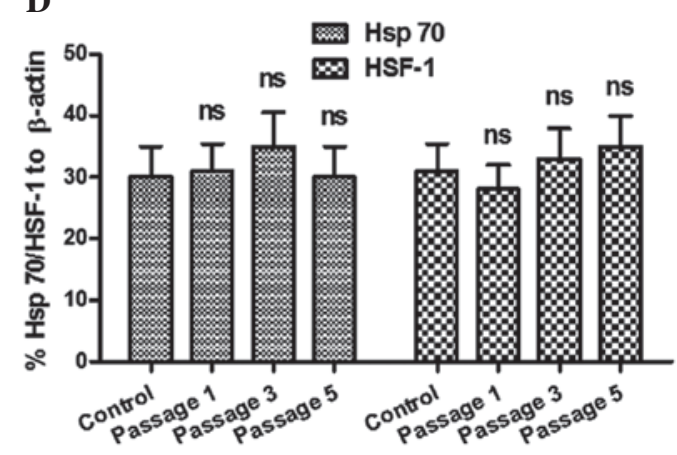

Figure 3. Expression of HSF-1 and Hsp70 in SH-SY5Y (Syn+) cells. (A) mRNA levels of $\alpha$-Syn, HSF-1 and Hsp70 in SH-SY5Y (Syn+) cells at various passages. (B) Western blot analysis of $\alpha$-Syn, HSF-1 and Hsp70 protein levels in SH-SY5Y (Syn+) cells at various passages. (C) Stable overexpression of $\alpha$-Syn protein in SH-SY5Y (Syn+) cells at various passages; (D) Protein expression of HSF-1 or Hsp70 was not dependent on $\alpha$-Syn overexpression. Normal/Control: CAT-pcDNA3.1(+)-transfected SH-SY5Y cells; passage 1, 3 or 5: SH-SY5Y (Syn+) cells which were propagated for 1, 3 or 5 passages. Values are expressed as the mean \pm standard deviation $(\mathrm{n}=3) .{ }^{* *} \mathrm{P}<0.01$. ns, no significance; SH-SY5Y (Syn+), $\alpha$-Syn-overexpressing SH-SY5Y cells; HSP, heat shock protein; HSF, heat shock factor; Gln, glutamine; $\alpha$-Syn, $\alpha$-synuclein. 

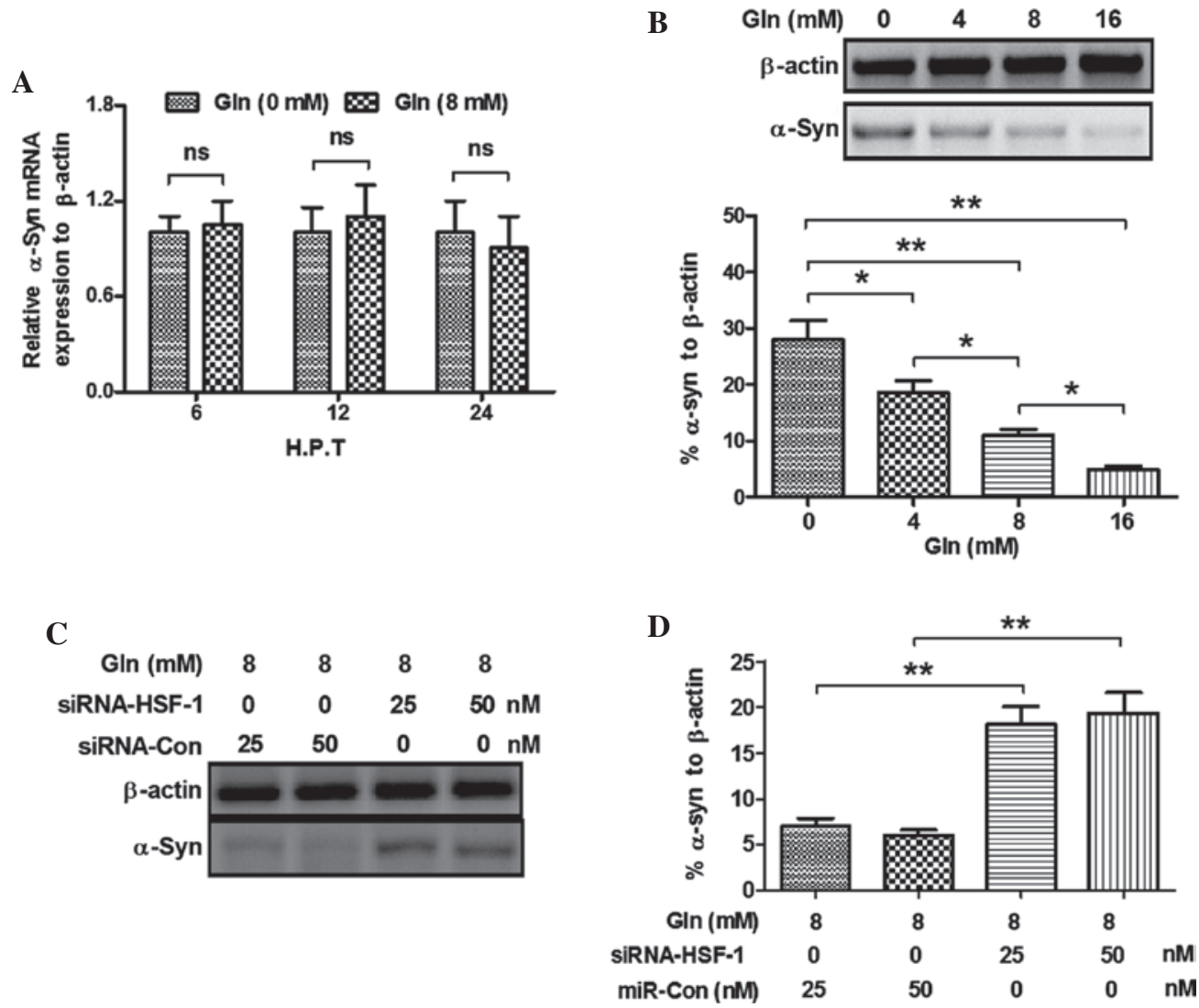

Figure 4. Gln-induced Hsp70 upregulation increases $\alpha$-Syn degradation. (A) mRNA levels of $\alpha$-Syn in the SH-SY5Y (Syn+) cells with or without 8 mM Gln treatment for 6,12 or $24 \mathrm{~h}$; (B) Western blot analysis indicated a significant reduction of $\alpha$-Syn levels the SH-SY5Y (Syn+) cells post-Gln treatment (4, 8 or $16 \mathrm{mM}$ for $24 \mathrm{~h}$ ); (C) Western blot analysis of $\alpha$-Syn levels in the SH-SY5Y (Syn+) cells post-Gln treatment and/or siRNA-HSF-1 transfection. (D) $\alpha$-Syn reduction by Gln treatment in the SH-SY5Y (Syn+) cells was abrogated by siRNA-HSF-1 transfection. All experiments were performed in triplicate. "P<0.05; ${ }^{* *} \mathrm{P}<0.01$. ns, no significance; siRNA, small interfering RNA; Con, control; SH-SY5Y (Syn+), $\alpha$-Syn-overexpressing SH-SY5Y cells; HSP, heat shock protein; HSF, heat shock factor; Gln, glutamine; $\alpha$-Syn, $\alpha$-synuclein.

present study constructed an SH-SY5Y cell clone which overexpressed wild-type $\alpha$-Syn, termed SH-SY5Y (Syn+). The coding sequence of wild-type $\alpha$-Synn was cloned into the eukaryotic expression vector, pcDNA3.1(+). The $\alpha$-Syn-overexpressing SH-SY5Y cell clone was selected using $800 \mu \mathrm{g} / \mathrm{ml} \mathrm{G} 418$ and maintained in complete medium containing $500 \mu \mathrm{g} / \mathrm{ml} \mathrm{G} 418$. Significantly higher and stably expressed levels of $\alpha$-Syn mRNA were detected in the SH-SY5Y (Syn+) cells following various passages $(\mathrm{P}<0.01 ;$ Fig. $3 \mathrm{~A})$. $\alpha$-Syn expression at the protein level was also significantly upregulated in the SH-SY5Y (Syn+) cells $(\mathrm{P}<0.01)$ (Fig. 3B and $\mathrm{C}$ ) according to western blot analysis. Furthermore, $\alpha$-Syn overexpression did not vary among various passages (Fig. 3A-D). To further investigate the influence of $\alpha$-Syn overexpression on Hsp70 and HSF-1, the mRNA expression of Hsp70 and HSF-1 was assessed by RT-qPCR and their protein levels by western blot analysis. Fig. 3A, B and D demonstrates that there was no difference in the mRNA and protein levels of Hsp70 and HSF-1 between SH-SY5Y cells transfected with the control CAT-pcDNA3.1(+) and SH-SY5Y (Syn+) cells, or among various passages of SH-SY5Y (Syn+) cells. Thus, the stably $\alpha$-Syn-overexpressing SH-SY5Y cells are suitable to be used for investigating the influence of Hsp70 and HSF-1 on $\alpha$-Syn degradation.

Upregulation of Hsp 70 by Gln increases $\alpha$-Syn degradation in SH-SY5Y (Syn+) cells. First, the possible regulation of $\alpha$-Syn expression in mRNA expression by Gln was investigated by RT-qPCR. Fig. 4A shows that $8 \mathrm{mM}$ Gln had no effect on $\alpha$-Syn mRNA levels in the SH-SY5Y (Syn+) cells at 6-12 h post-treatment. Furthermore, the protein levels of $\alpha$-Syn in SH-SY5Y (Syn+) cells post-Gln treatment were investigated. Fig. 4B demonstrates that Gln treatment for $24 \mathrm{~h}$ reduced the protein levels of $\alpha$-Syn $(\mathrm{P}<0.05$ for $4 \mathrm{mM}$ and $\mathrm{P}<0.01$ for 8 and $16 \mathrm{mM}$ ). A dose-dependence of the $\alpha$-Syn reduction was observed, as there was a significant difference between the 4and $8 \mathrm{mM}$ Gln groups $(\mathrm{P}<0.05)$ as well as between the 8 - and $16 \mathrm{mM}$ Gln groups $(\mathrm{P}<0.05)$ (Fig. 4B). In addition, the present study investigated the effect of HSF-1 knockdown on the $\alpha$-Syn reduction by Gln treatment. The results showed that 25 or $50 \mathrm{nM}$ siRNA-HIF-1 inhibited the $\alpha$-Syn reduction by Gln compared to that in cells transfected with the control siRNA $(\mathrm{P}<0.01$ for either concentration) (Fig. 4C and D). These results indicated that Gln treatment promoted $\alpha$-Syn degradation in SH-SY5Y (Syn+) cells, which was HSF-1-dependent.

\section{Discussion}

Accumulating evidence supports the key role of impaired $\alpha$-Syn degradation pathways $(18,19)$, followed by amyloid-like aggregation of $\alpha$-Syn, in PD $(32,33)$. The importance of molecular chaperones has been underlined by the fact that overexpression of these molecules, including HSPs, leads 
to re-folding of the aberrant $\alpha$-Syn aggregates to generate non-toxic and non-aggregated $\alpha$-Syn $(20,21)$. Therefore, functional defects of HSPs may have key roles in PD $(21,22)$, and promotion of HSP expression may be a potential strategy to prevent or ameliorate the aberrant $\alpha$-Syn aggregation, and thus control the progression of PD. Hsp70 is the most recognized molecular chaperone and has been linked with PD and $\alpha$-Syn aggregation. Substantial studies have confirmed the preventive role of Hsp70 in $\alpha$-Syn aggregation in PD $(22,23)$. Hsp70 is subject to transcriptional regulation upon various stresses and is regulated by a variety of molecules (34-36). Stress-inducible protein 1, an Hsp70/Hsp90-organizing protein, was confirmed to independently regulate the expression of Hsp70 (34); Parathyroid hormone activates adenylate cyclase and phospholipase C and subsequently promotes Hsp70 expression (35). Phorbol esters were also reported to deregulate the expression of Hsp70 and Hsp90 (36). Therefore, investigation of the deregulation of Hsp70 and its influence on $\alpha$-Syn degradation may shed light on the pathogenesis of PD.

HSF-1 has also been reported to be activated in response to chemical or thermal stresses and to promote the expression of HSPs, including Hsp70 (37-39). Following a cascade of post-translational modifications, including trimerization, nuclear translocation, DNA binding, and phosphorylation of its transactivation domain, activated HSF-1 binds to conserved regulatory sequences known as heat shock response elements and promotes HSP transcription $(40,41)$. Gln has been shown to safely enhance HSP expression in in vitro and in vivo settings (25-28). Gln mediates cellular protection against heat-stress injury to the lung via promoting HSF-1 expression, increasing HSF-1 promoter binding and phosphorylation, and then activating an HSP response (25). The protective effect of Gln was also confirmed in vivo, and was shown to proceed through the enhancement of HSF-1 phosphorylation/activation and promotion of HSP expression (26), particularly the promotion of Hsp70 expression (27). However, to the best of our knowledge, the protective effects of Gln against PD have not yet reported.

The present study reported the upregulation of Hsp70 expression by Gln in SH-SY5Y neuroblastoma cells. Gln treatment significantly upregulated Hsp70 expression at the mRNA and protein level in a dose-dependent and time-dependent manner. Given the key regulatory role of HSF-1 in Hsp70 expression, the effect of Gln on Hsp70 expression was re-evaluated following HSF-1 knockdown. It was shown that HSF-1-specific siRNA blocked HSF-1 expression at the mRNA and protein level, and this HSF-1 blockage blunted the upregulation of Hsp70 by Gln in the SH-SY5Y cells The results therefore confirmed that the Gln-induced Hsp70 upregulation was HSF-1-dependent. Furthermore, the present study demonstrated that the Gln-induced Hsp70 upregulation facilitated the degradation of $\alpha$-Syn, while it had no influence on $\alpha$-Syn mRNA levels in SH-SY5Y (Syn+) cells, implying a novel strategy for preventing the progression of $\mathrm{PD}$, which is thought to be caused by functional defects of HSPs, impairing the degradation of $\alpha$-Syn $(21,22)$.

In conclusion, the present study confirmed that Gln upregulated Hsp70 expression in SH-SY5Y neuroblastoma cells in an HSF-1-dependent manner. The upregulation of Hsp70 expression by glutamine increased the degradation of $\alpha$-Syn in $\alpha$-Syn-overexpressing SH-SY5Y cells. Therefore, Gln may be a potential therapeutic agent to prevent $\alpha$-Syn aggregation in PD. The use of Gln for the treatment and prevention of PD requires further investigation; in particular, Gln-mediated upregulation of HSP70 expression and $\alpha$-Syn degradation require validation in vivo.

\section{Acknowledgements}

The present study was supported by a grant from Renmin Hospital of Wuhan University (Wuhan, China).

\section{References}

1. Bertram L and Tanzi RE: The genetic epidemiology of neurodegenerative disease. J Clin Invest 115: 1449-1457, 2005

2. Wirdefeldt K, Adami HO, Cole P, Trichopoulos D and Mandel J: Epidemiology and etiology of Parkinson's disease: A review of the evidence. Eur J Epidemiol 26 Suppl 1: S1-S58, 2011.

3. Irizarry MC, Growdon W, Gomez-Isla T, Newell K, George JM, Clayton DF and Hyman BT: Nigral and cortical Lewy bodies and dystrophic nigral neurites in Parkinson's disease and cortical lewy body disease contain alpha-synuclein immunoreactivity. J Neuropathol Exp Neurol 57: 334-337, 1998.

4. Spillantini MG, Crowther RA, Jakes R, Hasegawa M and Goedert M: Alpha-Synuclein in filamentous inclusions of Lewy bodies from Parkinson's disease and dementia with lewy bodies. Proc Natl Acad Sci USA 95: 6469-6473, 1998.

5. Jellinger KA: Neuropathology of sporadic Parkinson's disease: Evaluation and changes of concepts. Mov Disord 27: 8-30, 2012.

6. Kruger R, Kuhn W, Muller T, Woitalla D, Graeber M, Kösel S, Przuntek H, Epplen JT, Schöls L and Riess O: Ala30Pro mutation in the gene encoding alpha-synuclein in Parkinson's disease. Nat Genet 18: 106-108, 1998.

7. Chartier-Harlin MC, Kachergus J, Roumier C, Mouroux V, Douay X, Lincoln S, Levecque C, Larvor L, Andrieux J, Hulihan M, et al: Alpha-synuclein locus duplication as a cause of familial Parkinson's disease. Lancet 364: 1167-1169, 2004.

8. Uéda K, Fukushima H, Masliah E, Xia Y, Iwai A, Yoshimoto M, Otero DA, Kondo J, Ihara Y and Saitoh T: Molecular cloning of cDNA encoding an unrecognized component of amyloid in Alzheimer disease. Proc Natl Acad Sci USA 90: 11282-11286, 1993.

9. Jakes R, Spillantini MG and Goedert M: Identification of two distinct synucleins from human brain. Febs Lett 345: 27-32, 1994.

10. Mueller A, Ziegler K, Amsharov KY and Jansen $M$ : Perchloropyracylene and its fusion with C60 by chlorine-assisted radio-frequency furnace synthesis. Chemistry 17: 11797-11804, 2011.

11. Bartels T, Choi JG and Selkoe DJ: $\alpha$-Synuclein occurs physiologically as a helically folded tetramer that resists aggregation. Nature 477: 107-110, 2011.

12. Chandra S, Gallardo G, Fernández-Chacón R, Schlüter OM and Südhof TC: Alpha-synuclein cooperates with CSPalpha in preventing neurodegeneration. Cell 123: 383-396, 2005.

13. Fauvet B, Mbefo MK, Fares MB, Desobry C, Michael S, Ardah MT, Tsika E, Coune P, Prudent M, Lion N, et al: $\alpha$-Synuclein in central nervous system and from erythrocytes, mammalian cells and Escherichia coli exists predominantly as disordered monomer. J Biol Chem 287: 15345-15364, 2012.

14. Eichner T and Radford SE: A diversity of assembly mechanisms of a generic amyloid fold. Mol Cell 43: 8-18, 2011.

15. Conway KA, Harper JD and Lansbury PT: Accelerated in vitro fibril formation by a mutant alpha-synuclein linked to early-onset Parkinson disease. Nat Med 4: 1318-1320, 1998.

16. Alvarez-Erviti L, Seow Y, Schapira AH, Rodriguez-Oroz MC, Obeso JA and Cooper JM: Influence of microRNA deregulation on chaperone-mediated autophagy and $\alpha$-synuclein pathology in Parkinson's disease. Cell Death Dis 4: e545, 2013.

17. Singleton AB, Farrer M, Johnson J, Singleton A, Hague S, Kachergus J, Hulihan M, Peuralinna T, Dutra A, Nussbaum R, et al: alpha-Synuclein locus triplication causes Parkinson's disease. Science 302: 841, 2003. 
18. Cuervo AM, Stefanis L, Fredenburg R, Lansbury PT and Sulzer D: Impaired degradation of mutant alpha-synuclein by chaperone-mediated autophagy. Science 305: 1292-1295, 2004

19. Alvarez-Erviti L, Rodriguez-Oroz MC, Cooper JM, Caballero C Ferrer I, Obeso JA and Schapira AH: Chaperone-mediated autophagy markers in Parkinson disease brains. Arch Neurol 67: 1464-1472, 2010

20. Periquet M, Fulga T, Myllykangas L, Schlossmacher MG and Feany MB: Aggregated alpha-synuclein mediates dopaminergic neurotoxicity in vivo. J Neurosci 27: 3338-3346, 2007.

21. Auluck PK, Chan HY, Trojanowski JQ, Lee VM and Bonini NM Chaperone suppression of alpha-synuclein toxicity in a Drosophila model for Parkinson's disease. Science 295: 865-868, 2002.

22. Klucken J, Shin Y, Masliah E, Hyman BT and McLean PJ Hsp70 reduces alpha-synuclein aggregation and toxicity. J Biol Chem 279: 25497-25502, 2004.

23. Luk KC, Mills IP, Trojanowski JQ and Lee VM: Interactions between Hsp70 and the hydrophobic core of alpha-synuclein inhibit fibril assembly. Biochemistry 47: 12614-12625, 2008.

24. Livak KJ and Schmittgen TD: Analysis of relative gene expression data using real-time quantitative PCR and the 2(-Delta Delta C(T)) Method. Methods 25: 402-408, 2001.

25. Morrison AL, Dinges M, Singleton KD, Odoms K, Wong HR and Wischmeyer PE: Glutamine's protection against cellular injury is dependent on heat shock factor-1. Am J Physiol Cell Physiol 290: C1625-C1632, 2006.

26. Singleton KD, Serkova N, Beckey VE and Wischmeyer PE: Glutamine attenuates lung injury and improves survival after sepsis: Role of enhanced heat shock protein expression. Crit Care Med 33: 1206-1213, 2005.

27. Singleton KD and Wischmeyer PE: Glutamine's protection against sepsis and lung injury is dependent on heat shock protein 70 expression. Am J Physiol Regul Integr Comp Physiol 292: R1839-R1845, 2007.

28. Wischmeyer PE, Kahana M, Wolfson R, Ren H, Musch MM and Chang EB: Glutamine induces heat shock protein and protects against endotoxin shock in the rat. J Appl Physiol (1985) 90: 2403-2410, 2001.
29. Pelham HR: A regulatory upstream promoter element in the Drosophila hsp 70 heat-shock gene. Cell 30: 517-528, 1982.

30. Amin J, Ananthan J and Voellmy R: Key features of heat shock regulatory elements. Mol Cell Biol 8: 3761-3769, 1988.

31. Sorger PK: Heat shock factor and the heat shock response. Cell 65: 363-366, 1991.

32. Gupta A, Dawson VL and Dawson TM: What causes cell death in Parkinson's disease? Ann Neurol (64 Suppl 2): S3-S15, 2008.

33. Jellinger KA: Basic mechanisms of neurodegeneration: A critical update. J Cell Mol Med 14: 457-487, 2010.

34. Song Y and Masison DC: Independent regulation of Hsp70 and Hsp90 chaperones by Hsp70/Hsp90-organizing protein Sti1 (Hop1). J Biol Chem 280: 34178-34185, 2005.

35. Fukayama S, Lanske B, Guo J, Kronenberg HM and Bringhurst FR: Regulation of HSP70 by PTH: A model of gene regulation not mediated by changes in cAMP levels. Am J Physiol 271: C121-C129, 1996.

36. Jacquier-Sarlin MR, Jornot L and Polla BS: Differential expression and regulation of hsp70 and hsp90 by phorbol esters and heat shock. J Biol Chem 270: 14094-14099, 1995.

37. Sorger PK: Heat shock factor and the heat shock response. Cell 65: 363-366, 1991.

38. Sarge KD, Murphy SP and Morimoto RI: Activation of heat shock gene transcription by heat shock factor 1 involves oligomerization, acquisition of DNA-binding activity and nuclear localization and can occur in the absence of stress. Mol Cell Biol 13: 1392-1407, 1993.

39. Cotto JJ, Kline M and Morimoto RI: Activation of heat shock factor 1 DNA binding precedes stress-induced serine phosphorylation. Evidence for a multistep pathway of regulation. J Biol Chem 271: 3355-3358, 1996.

40. Sasi BK, Sonawane PJ, Gupta V, Sahu BS and Mahapatra NR: Coordinated transcriptional regulation of Hspala gene by multiple transcription factors: Crucial roles for HSF-1, NF-Y, NF- $\kappa$ B and CREB. J Mol Biol 426: 116-135, 2014.

41. Tetievsky A and Horowitz M: Posttranslational modifications in histones underlie heat acclimation-mediated cytoprotective memory. J Appl Physiol (1985) 109: 1552-1561, 2010. 